\title{
Inversions in Split Trees and Conditional Galton-Watson Trees
}

\section{Xing Shi Cai}

Department of Mathematics, Uppsala University, Uppsala, Sweden xingshi.cai@math.uu.se

(D) https://orcid.org/0000-0002-3768-8078

\section{Cecilia Holmgren}

Department of Mathematics, Uppsala University, Uppsala, Sweden cecilia.holmgren@math.uu.se

(D) https://orcid.org/0000-0003-0717-4671

\section{Svante Janson}

Department of Mathematics, Uppsala University, Uppsala, Sweden svante.janson@math.uu.se

(D) https://orcid.org/0000-0002-9680-2790

\section{Tony Johansson}

Department of Mathematics, Uppsala University, Uppsala, Sweden tony.johansson@math.uu.se

\section{Fiona Skerman}

Department of Mathematics, Uppsala University, Uppsala, Sweden fiona.skerman@math.uu.se

(D) https://orcid.org/0000-0003-4141-7059

\begin{abstract}
We study $I(T)$, the number of inversions in a tree $T$ with its vertices labeled uniformly at random. We first show that the cumulants of $I(T)$ have explicit formulas. Then we consider $X_{n}$, the normalized version of $I\left(T_{n}\right)$, for a sequence of trees $T_{n}$. For fixed $T_{n}$ 's, we prove a sufficient condition for $X_{n}$ to converge in distribution. For $T_{n}$ being split trees [6], we show that $X_{n}$ converges to the unique solution of a distributional equation. Finally, when $T_{n}$ 's are conditional Galton-Watson trees, we show that $X_{n}$ converges to a random variable defined in terms of Brownian excursions. Our results generalize and extend previous work by Panholzer and Seitz [20].
\end{abstract}

2012 ACM Subject Classification Mathematics of computing $\rightarrow$ Probabilistic algorithms

Keywords and phrases inversions, random trees, split trees, Galton-Watson trees, permutation, cumulant

Digital Object Identifier 10.4230/LIPIcs.AofA.2018.15

Funding This work was partially supported by the Knut and Alice Wallenberg Foundation, the Swedish Research Council and the Ragnar Söderbergs Foundation.

Acknowledgements We want to thank Henning Sulzbach for helpful discussions.

(c) (i) $\odot$ Xing Shi Cai, Cecilia Holmgren, Svante Janson, Tony Johansson, and Fiona Skerman;

cc) licensed under Creative Commons License CC-BY

29th International Conference on Probabilistic, Combinatorial and Asymptotic Methods for the Analysis of Algorithms (AofA 2018).

Editors: James Allen Fill and Mark Daniel Ward; Article No. 15; pp. 15:1-15:12

Leibniz International Proceedings in Informatics

LIPICS Schloss Dagstuhl - Leibniz-Zentrum für Informatik, Dagstuhl Publishing, Germany 


\section{Introduction}

\subsection{Inversions in a fixed tree}

Let $\sigma_{1}, \ldots, \sigma_{n}$ be a permutation of $\{1, \ldots, n\}$. If $i<j$ and $\sigma_{i}>\sigma_{j}$, then the pair $\left(\sigma_{i}, \sigma_{j}\right)$ is called an inversion. The concept of inversions was introduced by Cramer [5] (1750) due to its connection with solving linear equations. More recently, the study of inversions has been motivated by its applications in the analysis of sorting algorithms ([15, Section 5.1]). Many authors, e.g., Feller [7, pp. 256], have shown that the number of inversions in uniform random permutations has a central limit theorem.

The concept of inversions can be generalized as follows. Consider an unlabeled rooted tree $T$ on node set $V$. Let $\rho$ denote the root. Write $u<v$ if $u$ is a proper ancestor of $v$, i.e., the unique path from $\rho$ to $v$ passes through $u$ and $u \neq v$. Write $u \leq v$ if $u$ is an ancestor of $v$, i.e., either $u<v$ or $u=v$. Given a bijection $\lambda: V \rightarrow\{1, \ldots,|V|\}$ (a node labeling), define the number of inversions

$$
I(T, \lambda) \stackrel{\text { def }}{=} \sum_{u<v} \mathbf{1}_{\lambda(u)>\lambda(v)} .
$$

Note that if $T$ is a path, then $I(T, \lambda)$ is nothing but the number of inversions in a permutation. Our main object of study is the random variable $I(T)$, defined by $I(T)=I(T, \lambda)$ where $\lambda$ is chosen uniformly at random from the set of bijections from $V$ to $\{1, \ldots,|V|\}$.

The enumeration of trees with a fixed number of inversions has been studied by Mallows and Riordan [16] and Gessel et al. [9] using the so called inversions polynomial. While analyzing linear probing hashing, Flajolet et al. [8] noticed that the numbers of inversions in Cayley trees with uniform random labeling converges to an Airy distribution. Panholzer and Seitz [20] showed that this is true for conditional Galton-Watson trees, which encompasses the case of Cayley trees.

For a node $v$, let $z_{v}$ denote the size of the subtree rooted at $v$. The following representation of $I(T)$ is the basis of most of our results:

- Lemma 1. Let $T$ be a fixed tree. Then

$$
I(T) \stackrel{\mathrm{d}}{=} \sum_{v \in V} Z_{v}
$$

where $\left\{Z_{v}\right\}_{v \in V}$ are independent random variables, and $Z_{v} \sim \operatorname{Unif}\left\{0,1, \ldots, z_{v}-1\right\}$.

We will generally be concerned with the centralized number of inversions, i.e., $I(T)-$ $\mathbb{E}[I(T)]$. For any $u<v$ we have $\mathbb{P}\{\lambda(u)>\lambda(v)\}=1 / 2$. Let $h(v)$ denote the depth of $v$, i.e., the distance from $v$ to the root $\rho$. It immediately follows that,

$$
\mathbb{E}[I(T)]=\sum_{u<v} \mathbb{E}\left[\mathbf{1}_{\lambda(u)>\lambda(v)}\right]=\frac{1}{2} \Upsilon(T),
$$

where $\Upsilon(T) \stackrel{\text { def }}{=} \sum_{v} h(v)$ is called the total path length (or internal path length) of $T$.

Let $\varkappa_{k}=\varkappa_{k}(X)$ denote the $k$-th cumulant of a random variable $X$ (provided it exists); thus $\varkappa_{1}(X)=\mathbb{E}[X]$ and $\varkappa_{2}(X)=\operatorname{Var}(X)$. We now define $\Upsilon_{k}(T)$, the $k$-total common ancestors of $T$, which allows us to generalize (1.1) to higher cumulants of $I(T)$. For $k$ nodes $v_{1}, \ldots, v_{k}$ (not necessarily distinct), let $c\left(v_{1}, \ldots, v_{k}\right)$ be the number of ancestors that they share, i.e.,

$$
c\left(v_{1}, \ldots, v_{k}\right) \stackrel{\text { def }}{=}\left|\left\{u \in V: u \leq v_{1}, u \leq v_{2}, \ldots, u \leq v_{k}\right\}\right| .
$$


We define

$$
\Upsilon_{k}(T) \stackrel{\text { def }}{=} \sum_{v_{1}, \ldots, v_{k}} c\left(v_{1}, \ldots, v_{k}\right),
$$

where the sum is over all ordered $k$-tuples of nodes in the tree. For a single node $v$, $h(v)=c(v)-1$, since $v$ itself is counted in $c(v)$. So $\Upsilon(T)=\Upsilon_{1}(T)-|V|$; i.e., we recover the usual notion of total path length. Using Lemma 1, it is easy to show the following:

- Theorem 2. Let $T$ be a fixed tree. Let $\varkappa_{k}(I(T))$ be the $k$-th cumulant of $I(T)$. Then

$$
\begin{aligned}
\mathbb{E}[I(T)] & =\varkappa_{1}(I(T))=\frac{1}{2} \Upsilon(T)=\frac{1}{2}\left(\Upsilon_{1}(T)-|V|\right), \\
\operatorname{Var}(I(T)) & =\varkappa_{2}(I(T))=\frac{1}{12}\left(\Upsilon_{2}(T)-|V|\right),
\end{aligned}
$$

and, more generally, for $k \geq 1$,

$$
\varkappa_{2 k+1}(I(T))=0, \quad \varkappa_{2 k}(I(T))=\frac{B_{2 k}}{2 k}\left(\Upsilon_{2 k}(T)-|V|\right),
$$

where $B_{k}$ denotes the $k$-th Bernoulli number. Moreover, $I(T)$ has the moment generating function

$$
\mathbb{E}\left[e^{t I(T)}\right]=\prod_{v \in V} \frac{e^{z_{v} t}-1}{z_{v}\left(e^{t}-1\right)},
$$

and for the centralized variable we have the estimate

$$
\mathbb{E}\left[e^{t(I(T)-\mathbb{E}[I(T)])}\right] \leq \exp \left(\frac{1}{8} t^{2} \sum_{v \in T}\left(z_{v}-1\right)^{2}\right) \leq \exp \left(\frac{1}{8} t^{2} \sum_{v \in T} z_{v}^{2}\right)=\exp \left(\frac{1}{8} t^{2} \Upsilon_{2}(T)\right), \quad t \in \mathbb{R}
$$

\subsection{Inversions in sequences of trees}

The total path length $\Upsilon(T)$ has been studied for random trees like split trees [3] and conditional Galton-Watson trees [1, Corollary 9]. This leads us to focus on the deviation

$$
X_{n}=\frac{I\left(T_{n}\right)-\mathbb{E}\left[I\left(T_{n}\right)\right]}{s(n)},
$$

under some appropriate scaling $s(n)$, for a sequence of (random or fixed) trees $T_{n}$.

\section{Fixed trees}

The following theorem follows easily from Theorem 2:

- Theorem 3. Let $T_{n}$ be a sequence of fixed trees on $n$ nodes. Let

$$
X_{n}=\frac{I\left(T_{n}\right)-\mathbb{E}\left[I\left(T_{n}\right)\right]}{\sqrt{\Upsilon_{2}\left(T_{n}\right)}} .
$$

Assume that for all $k \geq 1$,

$$
\frac{\Upsilon_{2 k}\left(T_{n}\right)}{\Upsilon_{2}\left(T_{n}\right)^{k}} \rightarrow \zeta_{2 k}
$$

for some sequence $\left(\zeta_{2 k}\right)$. Then there exists a unique distribution $X$ with

$$
\varkappa_{2 k-1}(X)=0, \quad \varkappa_{2 k}(X)=\frac{B_{2 k}}{2 k} \zeta_{2 k}, \quad k \geq 1,
$$

such that $X_{n} \stackrel{\mathrm{d}}{\longrightarrow} X$ and, moreover, $\mathbb{E}\left[e^{t X_{n}}\right] \rightarrow \mathbb{E}\left[e^{t X}\right]<\infty$ for every $t \in \mathbb{R}$. 
- Example 4. When $P_{n}$ is a path of $n$ nodes, we have for fixed $k \geq 1$

$$
\Upsilon_{k}\left(P_{n}\right) \sim \frac{1}{k+1} n^{k+1} .
$$

Thus $\Upsilon_{2 k}\left(P_{n}\right) / \Upsilon_{2}\left(P_{n}\right)^{k} \rightarrow \varkappa_{2 k}=0$ for $k \geq 2$. So by Theorem $3, X_{n}$ converges to a normal distribution, and we recover the central limit law for inversions in permutations. Also, the vertices have subtree sizes $1, \ldots, n$ and so we also recover from Theorem 2 the moment generating function $\prod_{j=1}^{n}\left(e^{j t}-1\right) /\left(j\left(e^{t}-1\right)\right)[22,17]$.

Other examples where Theorem 2 can be easily applied include complete $b$-ary trees and stars (a star is a tree containing only a root and leaves).

\section{Random trees}

We move on to random trees. We consider generating a random tree $T_{n}$ and, conditioning on $T_{n}$, labeling its nodes uniformly at random. The relation (1.1) is maintained for random trees:

$$
\mathbb{E}\left[I\left(T_{n}\right)\right]=\mathbb{E}\left[\mathbb{E}\left[I\left(T_{n}\right) \mid T_{n}\right]\right]=\frac{1}{2} \mathbb{E}\left[\Upsilon\left(T_{n}\right)\right] .
$$

The deviation of $I\left(T_{n}\right)$ from its mean can be taken to mean two different things. Consider for some scaling function $s(n)$,

$$
X_{n}=\frac{I\left(T_{n}\right)-\mathbb{E}\left[I\left(T_{n}\right)\right]}{s(n)}, \quad Y_{n}=\frac{I\left(T_{n}\right)-\mathbb{E}\left[I\left(T_{n}\right) \mid T_{n}\right]}{s(n)}=\frac{I\left(T_{n}\right)-\frac{1}{2} \Upsilon\left(T_{n}\right)}{s(n)} .
$$

Then $X_{n}$ and $Y_{n}$ each measure the deviation of $I\left(T_{n}\right)$, unconditionally and conditionally. They are related by the identity

$$
X_{n}=Y_{n}+W_{n} / 2
$$

where

$$
W_{n}=\frac{\Upsilon\left(T_{n}\right)-\mathbb{E}\left[\Upsilon\left(T_{n}\right)\right]}{s(n)} .
$$

In the case of fixed trees $W_{n}=0$ and $X_{n}=Y_{n}$, but for random trees we consider the sequences separately.

\section{Split trees}

The first class of random trees which we study are split trees. They were introduced by Devroye [6] to encompass many families of trees that are frequently used in algorithm analysis, e.g., binary search trees, $m$-ary search trees, digital search trees, etc.

A split tree can be constructed as follows. Consider a rooted infinite $b$-ary tree where each node is a bucket of finite capacity $s$. We place $n$ balls at the root, and the balls individually trickle down the tree in a random fashion until no bucket is above capacity. Each node draws a split vector $\mathcal{V}=\left(V_{1}, \ldots, V_{b}\right)$ from a common distribution, where $V_{i}$ describes the probability that a ball passing through the node continues to the $i$ th child. The trickle-down procedure is defined precisely in Section 2. Any node $u$ such that the subtree rooted at $u$ contains no balls is then removed, and we consider the resulting tree $T_{n}$.

In the context of split trees we differentiate between $I\left(T_{n}\right)$ (the number of inversions on nodes), and $\hat{I}\left(T_{n}\right)$ (the number of inversions on balls). In the former case, the nodes (buckets) 
are given labels, while in the latter the individual balls are given labels. For balls $\beta_{1}, \beta_{2}$, write $\beta_{1}<\beta_{2}$ if the node containing $\beta_{1}$ is a proper ancestor of the node containing $\beta_{2}$; if $\beta_{1}, \beta_{2}$ are contained in the same node we do not compare their labels. Define

$$
\hat{I}\left(T_{n}\right)=\sum_{\beta_{1}<\beta_{2}} \mathbf{1}_{\lambda\left(\beta_{1}\right)>\lambda\left(\beta_{2}\right)} .
$$

Similarly define $\hat{\Upsilon}\left(T_{n}\right)$ as the total path length on balls, i.e., the sum of the depth of all balls. And let

$$
\hat{X}_{n}=\frac{\hat{I}\left(T_{n}\right)-\mathbb{E}\left[\hat{I}\left(T_{n}\right)\right]}{n}, \quad \hat{Y}_{n}=\frac{\hat{I}\left(T_{n}\right)-s_{0} \hat{\Upsilon}\left(T_{n}\right) / 2}{n}, \quad \hat{W}_{n}=\frac{\hat{\Upsilon}\left(T_{n}\right)-\mathbb{E}\left[\hat{\Upsilon}\left(T_{n}\right)\right]}{n} .
$$

Here $s_{0}$ is a fixed integer denoting the number of balls in any internal node, and we have $\hat{X}_{n}=\hat{Y}_{n}+s_{0} \hat{W}_{n} / 2$ (justified in Section 2). The following theorem gives the limiting distributions of the random vector $\left(\hat{X}_{n}, \hat{Y}_{n}, \hat{W}_{n}\right)$. In a longer version of this paper [4], we also have a similar result for $\left(X_{n}, Y_{n}, W_{n}\right)$ under stronger assumptions. Note that the concepts are identical for any class of split trees where each node holds exactly one ball, such as binary search trees and digital search trees.

Let $d_{2}$ denote the Mallows metric, also called the minimal $\ell_{2}$ metric (defined in Section 2 ). Let $\mathcal{M}_{0,2}^{d}$ be the set of probability measures on $\mathbb{R}^{d}$ with zero mean and finite second moment.

- Theorem 5. Let $T_{n}$ be a split tree and let $\mathcal{V}=\left(V_{1}, \ldots, V_{b}\right)$ be a split vector. Define

$$
\mu=-\sum_{i=1}^{b} \mathbb{E}\left[V_{i} \ln V_{i}\right], \quad \text { and } \quad D(\mathcal{V})=\frac{1}{\mu} \sum_{i=1}^{b} V_{i} \ln V_{i}
$$

Assume that $\mathbb{P}\left\{\exists i: V_{i}=1\right\}<1$ and $s_{0}>0$. Let $(\hat{X}, \hat{Y}, \hat{W})$ be the unique solution in $\mathcal{M}_{0,2}^{3}$ for the system of fixed-point equations

$$
\left[\begin{array}{l}
\hat{X} \\
\hat{Y} \\
\hat{W}
\end{array}\right] \stackrel{\mathrm{d}}{=}\left[\begin{array}{l}
\sum_{i=1}^{b} V_{i} \hat{X}^{(i)}+\sum_{j=1}^{s_{0}} U_{j}+\frac{s_{0}}{2} D(\mathcal{V}) \\
\sum_{i=1}^{b} V_{i} \hat{Y}^{(i)}+\sum_{j=1}^{s_{0}}\left(U_{j}-1 / 2\right) \\
\sum_{i=1}^{b} V_{i} \hat{W}^{(i)}+1+D(\mathcal{V})
\end{array}\right] .
$$

Here $\left(V_{1}, \ldots, V_{b}\right), U_{1}, \ldots, U_{s_{0}},\left(\hat{X}^{(1)}, \hat{Y}^{(1)}, \hat{W}^{(1)}\right), \ldots,\left(\hat{X}^{(b)}, \hat{Y}^{(b)}, \hat{W}^{(b)}\right)$ are independent, with $U_{j} \sim \operatorname{Unif}[0,1]$ for $j=1, \ldots, s_{0}$, and $\left(\hat{X}_{n}^{(i)}, \hat{Y}_{n}^{(i)}, \hat{W}_{n}^{(i)}\right) \sim(\hat{X}, \hat{Y}, \hat{W})$ for $i=1, \ldots, b$. Then the sequence $\left(\hat{X}_{n}, \hat{Y}_{n}, \hat{W}_{n}\right)$ defined in (1.3) converges to $(\hat{X}, \hat{Y}, \hat{W})$ in $d_{2}$ and in moment generating function within a neighborhood of the origin.

The proof of Theorem 5 uses the contraction method, introduced by Rösler [21] for finding the total path length of binary search trees. The technique has been applied to $d$-dimensional quad trees by Neininger and Rüschendorf [19] and to split trees in general by Broutin and Holmgren [3]. 


\section{Conditional Galton-Watson trees}

A conditional Galton-Watson tree $T_{n}$ is a Galton-Watson tree conditioned on having $n$ nodes, which we define in details in Section 3. It generalizes many uniform random tree models, e.g., Cayley trees, Catalan trees, binary trees, $b$-ary trees, and Motzkin trees. For a comprehensive survey, see Janson [12].

Aldous [1] showed that many asymptotic properties of conditional Galton-Watson trees, such as the height and the total path length, can be derived from properties of Brownian excursions. Our analysis of inversions follows a similar route. In particular, we relate $I\left(T_{n}\right)$ to the Brownian snake studied by e.g., Janson and Marckert [14].

In the context of Galton-Watson trees, Aldous [1, Corollary 9] showed that $n^{-3 / 2} \Upsilon\left(T_{n}\right)$ converges to an Airy distribution. We will see that the standard deviation of $I\left(T_{n}\right)-\frac{1}{2} \Upsilon\left(T_{n}\right)$ is of order $n^{5 / 4} \ll n^{3 / 2}$, which by the decomposition (1.2) implies that $n^{-3 / 2} I\left(T_{n}\right)$ converges to the same Airy distribution, recovering one of the main results of Panholzer and Seitz [20, Theorem 5.3]. Our contribution for conditional Galton-Watson trees is a detailed analysis of $Y_{n}$ under the scaling function $s(n)=n^{5 / 4}$.

Let $e(s), s \in[0,1]$ be the random path of a standard Brownian excursion, and define $C(s, t) \stackrel{\text { def }}{=} C(t, s) \stackrel{\text { def }}{=} 2 \min _{s \leq u \leq t} e(u)$ for $0 \leq s \leq t \leq 1$.

We define a random variable, see [11],

$$
\eta \stackrel{\text { def }}{=} \int_{[0,1]^{2}} C(s, t) \mathrm{d} s \mathrm{~d} t=4 \int_{0 \leq s \leq t \leq 1} \min _{s \leq u \leq t} e(u) .
$$

- Theorem 6. Suppose $T_{n}$ is a conditional Galton-Watson tree with offspring distribution $\xi$ such that $\mathbb{E}[\xi]=1$, $\operatorname{Var}(\xi)=\sigma^{2} \in(0, \infty)$, and $\mathbb{E}\left[e^{\alpha \xi}\right]<\infty$ for some $\alpha>0$, and define

$$
Y_{n}=\frac{I\left(T_{n}\right)-\frac{1}{2} \Upsilon\left(T_{n}\right)}{n^{5 / 4}}
$$

Then we have

$$
Y_{n} \stackrel{\mathrm{d}}{\longrightarrow} Y \stackrel{\text { def }}{=} \frac{1}{\sqrt{12 \sigma}} \sqrt{\eta} \mathcal{N}
$$

where $\mathcal{N}$ is a standard normal random variable, independent from the random variable $\eta$ defined in (1.5). Moreover, $\mathbb{E}\left[e^{t Y_{n}}\right] \rightarrow \mathbb{E}\left[e^{t Y}\right]<\infty$ for all fixed $t \in \mathbb{R}$.

In the rest of the paper, we outline the proofs of our main results, Theorem 5 and 6 . The proofs of Theorem 2 and 3 are omitted. The details of the proofs can be found in the longer version of this paper [4].

\section{A sequence of split trees}

In this section we outline how one can apply the contraction method to prove Theorem 5 .

We will now define split trees introduced by Devroye [6]. The random split tree $T_{n}$ has parameters $b, s, s_{0}, s_{1}, \mathcal{V}$ and $n$. The integers $b, s, s_{0}, s_{1}$ are required to satisfy the inequalities

$$
2 \leq b, \quad 0<s, \quad 0 \leq s_{0} \leq s, \quad 0 \leq b s_{1} \leq s+1-s_{0}
$$

and $\mathcal{V}=\left(V_{1}, \ldots, V_{b}\right)$ is a random non-negative vector with $\sum_{i=1}^{b} V_{i}=1$. Consider an infinite $b$-ary tree $\mathcal{U}$. The split tree $T_{n}$ is constructed by distributing $n$ balls (pieces of information) among nodes of $\mathcal{U}$. For a node $u$, let $n_{u}$ be the number of balls stored in the subtree rooted at $u$. Once $n_{u}$ are all decided, we take $T_{n}$ to be the largest subtree of $\mathcal{U}$ such that $n_{u}>0$ 
for all $u \in T_{n}$. Let the split vector $\mathcal{V} \in[0,1]^{b}$ be as before. Let $\mathcal{V}_{u}=\left(V_{u, 1}, \ldots, V_{u, b}\right)$ be the independent copy of $\mathcal{V}$ assigned to $u$. Let $u_{1}, \ldots, u_{b}$ be the child nodes of $u$. Conditioning on $n_{u}$ and $\mathcal{V}_{u}$, if $n_{u} \leq s$, then $n_{u_{i}}=0$ for all $i$; if $n_{u}>s$, then

$$
\left(n_{u_{1}}, \ldots, n_{u_{b}}\right) \sim \operatorname{Mult}\left(n-s_{0}-b s_{1}, V_{u, 1}, \ldots, V_{u, b}\right)+\left(s_{1}, s_{1}, \ldots, s_{1}\right),
$$

where Mult denotes multinomial distribution, and $b, s, s_{0}, s_{1}$ are integers satisfying (2.1). Note that $\sum_{i=1}^{b} n_{u_{i}} \leq n$ (hence the "splitting"). Naturally for the root $\rho, n_{\rho}=n$. Thus the distribution of $\left(n_{u}, \mathcal{V}_{u}\right)_{u \in V(\mathcal{U})}$ is completely defined.

Once all $n$ balls have been placed in $\mathcal{U}$, we obtain $T_{n}$ by deleting all nodes $u$ such that the subtree rooted at $u$ contains no balls. Note that an internal node of $T_{n}$ contains exactly $s_{0}$ balls, while a leaf contains a random amount in $\{1, \ldots, s\}$. We assume, as previous authors, that $\mathbb{P}\left\{\exists i: V_{i}=1\right\}<1$. We can assume that $\mathcal{V}$ has a permutation invariant distribution without loss of generality, since a uniform random permutation of subtree order does not change the number of inversions.

\subsection{Outline}

Recall that in (1.3), we define $\hat{X}_{n}, \hat{Y}_{n}$ and $\hat{W}_{n}$. Let $\bar{n}=\left(n_{1}, \ldots, n_{b}\right)$ denote the vector of the (random) number of balls in each of the $b$ subtrees of the root. Broutin and Holmgren [3] showed that, conditioning on $\bar{n}$,

$$
\hat{W}_{n} \stackrel{\mathrm{d}}{=} \sum_{i=1}^{b} \frac{n_{i}}{n} \hat{W}_{n_{i}}+\frac{n-s_{0}}{n}+\hat{D}_{n}(\bar{n}), \quad \hat{D}_{n}(\bar{n}) \stackrel{\text { def }}{=}-\frac{\mathbb{E}\left[\hat{\Upsilon}\left(T_{n}\right)\right]}{n}+\sum_{i=1}^{b} \frac{\mathbb{E}\left[\hat{\Upsilon}\left(T_{n_{i}}\right)\right]}{n} .
$$

We derive similar recursions for $\hat{X}_{n}$ and $\hat{Y}_{n}$. Conditioning on $\bar{n}, \hat{I}\left(T_{n}\right)$ satisfies the recursion

$$
\hat{I}\left(T_{n}\right) \stackrel{\mathrm{d}}{=} \hat{Z}_{\rho}+\sum_{i=1}^{b} \hat{I}\left(T_{n_{i}}\right)
$$

where $\hat{Z}_{\rho}$ denotes the number of inversions involving balls contained in the root $\rho$. Therefore, still conditioning on $\bar{n}$, we have

$$
\begin{aligned}
\hat{X}_{n} & \stackrel{\mathrm{d}}{=} \sum_{i=1}^{b} \frac{n_{i}}{n} \hat{X}_{n_{i}}+\frac{\hat{Z}_{\rho}}{n}-\frac{\mathbb{E}\left[\hat{I}\left(T_{n}\right)\right]}{n}+\sum_{i=1}^{b} \frac{\mathbb{E}\left[\hat{I}\left(T_{n_{i}}\right)\right]}{n} \\
& =\sum_{i=1}^{b} \frac{n_{i}}{n} \hat{X}_{n_{i}}+\frac{\hat{Z}_{\rho}}{n}-\frac{s_{0}}{2} \frac{\mathbb{E}\left[\hat{\Upsilon}\left(T_{n}\right)\right]}{n}+\frac{s_{0}}{2} \sum_{i=1}^{b} \frac{\mathbb{E}\left[\hat{\Upsilon}\left(T_{n_{i}}\right)\right]}{n} \\
& =\sum_{i=1}^{b} \frac{n_{i}}{n} \hat{X}_{n_{i}}+\frac{\hat{Z}_{\rho}}{n}+\frac{s_{0}}{2} \hat{D}_{n}(\bar{n}),
\end{aligned}
$$

where we use that

$$
\mathbb{E}\left[\hat{I}\left(T_{n}\right) \mid T_{n}\right]=\frac{s_{0}}{2} \hat{\Upsilon}\left(T_{n}\right) .
$$

It follows also from $(2.2)$ that $\hat{X}_{n}=\hat{Y}_{n}+\frac{s_{0}}{2} \hat{W}_{n}$ and

$$
\hat{Y}_{n} \stackrel{\mathrm{d}}{=} \sum_{i=1}^{b} \frac{n_{i}}{n} \hat{Y}_{n_{i}}+\frac{\hat{Z}_{\rho}}{n}-\frac{s_{0}}{2} \frac{n-s_{0}}{n} .
$$


It is not difficult to see that

$$
\frac{\hat{Z}_{\rho}}{n} \stackrel{L^{2}}{\longrightarrow} U_{1}+\cdots+U_{s_{0}}
$$

where $U_{1}, \ldots, U_{s_{0}}$ are independent and uniformly distributed in $[0,1]$. Broutin and Holmgren [3] have shown that $\hat{D}_{n}(\bar{n}) \stackrel{\text { a.s. }}{\longrightarrow} D(\mathcal{V})$, where

$$
\mu=-\sum_{i=1}^{b} \mathbb{E}\left[V_{i} \ln V_{i}\right], \quad \text { and } \quad D(\mathcal{V})=\frac{1}{\mu} \sum_{i=1}^{b} V_{i} \ln V_{i} .
$$

Together with $\left(n_{1} / n, \ldots, n_{b} / n\right) \stackrel{\text { a.s. }}{\longrightarrow}\left(V_{1}, \ldots, V_{b}\right)$ (by the law of large number), we arrive at the fixed-point equations (1.4) presented in Theorem 5.

For a random vector $X \in \mathbb{R}^{d}$, let $\|X\|$ be the Euclidean norm of $X$. Let $\|X\|_{2} \stackrel{\text { def }}{=} \sqrt{\mathbb{E}\left[\|X\|^{2}\right]}$. Recall that $\mathcal{M}_{0,2}^{d}$ denotes the set of probability measures on $\mathbb{R}^{d}$ with zero mean and finite second moment. The Mallows metric on $\mathcal{M}_{0,2}^{d}$ is defined by

$$
d_{2}(\nu, \lambda)=\inf \left\{\|X-Y\|_{2}: X \sim \lambda, Y \sim \nu\right\} .
$$

Using the contraction method, Broutin and Holmgren [3] proved that $\hat{W}_{n} \stackrel{d_{2}}{\longrightarrow} \hat{W}$, the unique solution of the last equation of (1.4) in $\mathcal{M}_{0,2}^{1}$.

We can apply the same contraction method to show that the vector $\left(\hat{X}_{n}, \hat{Y}_{n}, \hat{W}_{n}\right) \stackrel{d_{2}}{\longrightarrow}$ $(\hat{X}, \hat{Y}, \hat{W})$, the unique solution of (1.4) in $\mathcal{M}_{0,2}^{3}$. Assume that the independent vectors $\left(\hat{X}^{(i)}, \hat{Y}^{(i)}, \hat{W}^{(i)}\right), i=1, \ldots, b$ share some common distribution $\mu \in \mathcal{M}_{0,2}^{3}$. Let $F(\mu) \in \mathcal{M}_{0,2}^{3}$ be the distribution of the random vector given by the right hand side of (1.4). Using a coupling argument, we can show that for all $\nu, \lambda \in \mathcal{M}_{0,2}^{3}$,

$$
d_{2}(F(\nu), F(\lambda))<c d_{2}(\nu, \lambda)
$$

where $c \in(0,1)$ is a constant. Thus $F$ is a contraction and by Banach's fixed point theorem, (1.4) must have a unique solution $(\hat{X}, \hat{Y}, \hat{W}) \in \mathcal{M}_{0,2}^{3}$. Finally, we can use a similar coupling argument to show that $\left(\hat{X}_{n}, \hat{Y}_{n}, \hat{W}_{n}\right) \stackrel{d_{2}}{\longrightarrow}(\hat{X}, \hat{Y}, \hat{W})$.

Note that in [4], instead of carrying out the above argument in details, we actually used a result by Neininger [18] which gives us a shortcut.

\section{A sequence of conditional Galton-Watson trees}

Let $\xi$ be a random variable with $\mathbb{E}[\xi]=1$, Var $\xi=\sigma^{2}<\infty$, and $\mathbb{E}\left[e^{\alpha \xi}\right]<\infty$ for some $\alpha>0$, (The last condition is used in the proof below, but is presumably not necessary.) Let $G^{\xi}$ be a (possibly infinite) Galton-Watson tree with offspring distribution $\xi$. The conditional Galton-Watson tree $T_{n}^{\xi}$ on $n$ nodes is given by

$$
\mathbb{P}\left\{T_{n}^{\xi}=T\right\}=\mathbb{P}\left\{G^{\xi}=T \mid G^{\xi} \text { has } n \text { nodes }\right\}
$$

for any rooted tree $T$ on $n$ nodes. The assumption $\mathbb{E}[\xi]=1$ is justified by noting that if $\zeta$ is such that $\mathbb{P}\{\xi=i\}=c \theta^{i} \mathbb{P}\{\zeta=i\}$ for all $i \geq 0$ then $T_{n}^{\xi}$ and $T_{n}^{\zeta}$ are identically distributed; hence it is typically possible to replace an offspring distribution $\zeta$ by an equivalent one with mean 1 , see [12, Sec. 4].

We fix some $\xi$ and drop it from the notation, writing $T_{n}=T_{n}^{\xi}$. 
In a fixed tree $T$ with root $\rho$ and $n$ total nodes, for each node $v \neq \rho$ let $Q_{v} \sim$ $\operatorname{Unif}(-1 / 2,1 / 2)$, all independent, and let $Q_{\rho}=0$. For each node $v$ define

$$
\Phi_{v} \stackrel{\text { def }}{=} \sum_{u \leq v} Q_{u}, \quad \text { and let } \quad J(T) \stackrel{\text { def }}{=} \sum_{v \in T} \Phi_{v} .
$$

In other words, $\Phi_{u}$ is the sum of $Q_{v}$ for all $v$ on the path from the root to $u$. For each $v \neq \rho$ also define $Z_{v}=\left\lfloor\left(Q_{v}+1 / 2\right) z_{v}\right\rfloor$, where $z_{v}$ denotes the size of the subtree rooted at $v$. Then $Z_{v}$ is uniform in $\left\{0,1, \ldots, z_{v}-1\right\}$, and by Lemma 1 , the quantity

$$
I^{*}(T) \stackrel{\text { def }}{=} \sum_{v \neq \rho}\left(Z_{v}-\mathbb{E}\left[Z_{v}\right]\right)
$$

is equal in distribution to the centralized number of inversions in the tree $T$, ignoring inversions involving $\rho$. The main part (1.6) of Theorem 6 will follow from arguing that for a conditional Galton-Watson tree $T_{n}$,

$$
\frac{J\left(T_{n}\right)}{n^{5 / 4}} \stackrel{\mathrm{d}}{\longrightarrow} Y \stackrel{\text { def }}{=} \frac{1}{\sqrt{12 \sigma}} \sqrt{\eta} \mathcal{N} .
$$

Indeed, under the coupling of $Q_{v}$ and $Z_{v}$ above,

$$
\begin{aligned}
J\left(T_{n}\right) & =\sum_{v} \Phi_{v}=\sum_{v} \sum_{u: u \leq v} Q_{u}=\sum_{u} Q_{u} \sum_{v: u \leq v} 1=\sum_{u} Q_{u} z_{u} \\
& \leq \sum_{u \neq \rho}\left(Z_{u}-\frac{z_{u}}{2}+1\right)<n+I^{*}\left(T_{n}\right),
\end{aligned}
$$

and similarly $J\left(T_{n}\right)>I^{*}\left(T_{n}\right)-n$. As $\rho$ contributes at most $n$ inversions to $I\left(T_{n}\right)$, it follows from the triangle inequality that $\left|J\left(T_{n}\right)-\left(I\left(T_{n}\right)-\Upsilon\left(T_{n}\right) / 2\right)\right| \leq 2 n=o\left(n^{5 / 4}\right)$. Thus (3.1), once proved, will imply that

$$
Y_{n} \stackrel{\text { def }}{=} \frac{I\left(T_{n}\right)-\Upsilon\left(T_{n}\right) / 2}{n^{5 / 4}}=o(1)+\frac{J\left(T_{n}\right)}{n^{5 / 4}} \stackrel{\mathrm{d}}{\longrightarrow} Y .
$$

The quantity $J\left(T_{n}\right)$ and the limiting distribution (3.1) have been considered by several authors. In the interest of keeping this section self-contained, we will now outline the proof of (3.1) which relies on the concept of a discrete snake, a random curve which under proper rescaling converges to a Brownian snake, a curve related to a standard Brownian excursion. This convergence was shown by Gittenberger [10], and later in more generality by Janson and Marckert [14], whose notation we use.

Define $f:\{0, \ldots, 2(n-1)\} \rightarrow V$ by saying that $f(i)$ is the location of a depth-first search (under some fixed ordering of nodes) at stage $i$, with $f(0)=f(2(n-1))=\rho$. Also define $V_{n}(i)=d(\rho, f(i))$ where $d$ denotes distance. The process $V_{n}(i)$ is called the depth-first walk, the Harris walk or the tour of $T_{n}$. For non-integer values $t, V_{n}(t)$ is given by linearly interpolating adjacent values. See Figure 1.

Finally, define $R_{n}(i) \stackrel{\text { def }}{=} \Phi_{f(i)}$ to be the value at the vertex visited after $i$ steps. For non-integer values $t, R_{n}(t)$ is defined by linearly interpolating the integer values. Also define $\widetilde{R}_{n}(t)$ by $\widetilde{R}_{n}(t) \stackrel{\text { def }}{=} R_{n}(t)$ when $t \in\{0,1, \ldots, 2 n\}$, and

$$
\widetilde{R}_{n}(t) \stackrel{\text { def }}{=}\left\{\begin{array}{lll}
R_{n}(\lfloor t\rfloor), & \text { if } & V_{n}(\lfloor t\rfloor)>V_{n}(\lceil t\rceil), \\
R_{n}(\lceil t\rceil), & \text { if } & V_{n}(\lfloor t\rfloor)<V_{n}(\lceil t\rceil) .
\end{array}\right.
$$



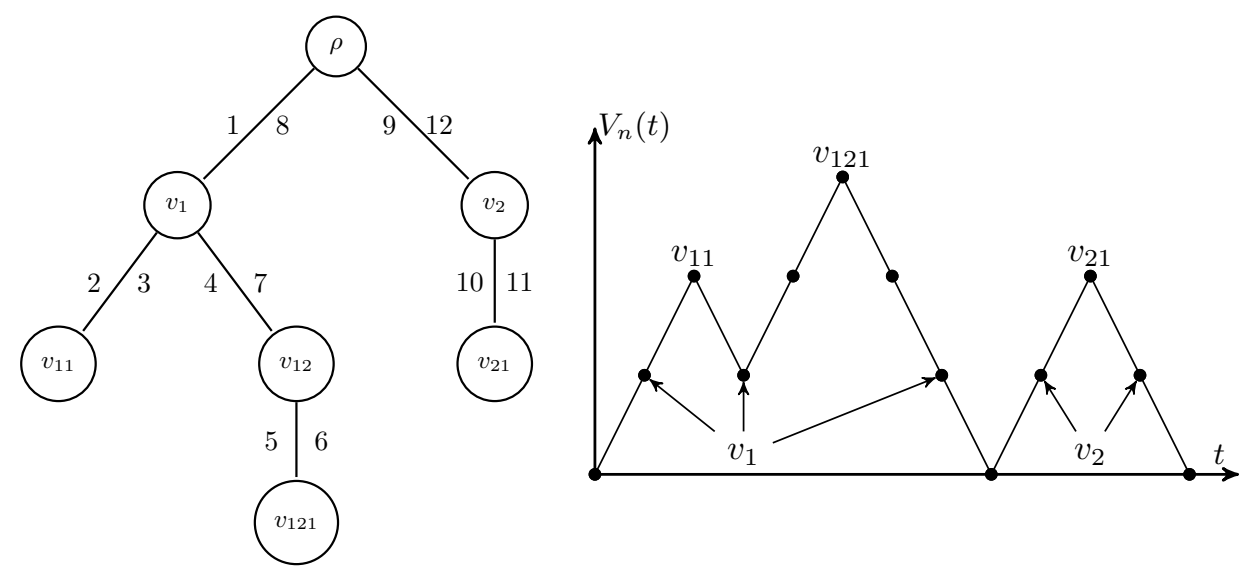

Figure 1 The depth-first walk $V_{n}(t)$ of a fixed tree.

In other words, $\widetilde{R}_{n}(t)$ takes the value of node $f(\lfloor t\rfloor)$ or $f(\lceil t\rceil)$, whichever is further from the root. We can recover $J\left(T_{n}\right)$ from $\widetilde{R}_{n}(t)$ via

$$
2 J\left(T_{n}\right)=\int_{0}^{2(n-1)} \widetilde{R}_{n}(t) d t
$$

Indeed, for each non-root node $v$ there are precisely two unit intervals during which $\widetilde{R}_{n}(t)$ draws its value from $v$, namely the two unit intervals during which the parent edge of $v$ is being traversed. Now, since $Q_{v} \sim \operatorname{Unif}(-1 / 2,1 / 2)$ we have $\left|R_{n}(i)-R_{n}(i-1)\right| \leq 1 / 2$ for all $i>0$ and

$$
\frac{J\left(T_{n}\right)}{n^{5 / 4}}=\frac{1}{2 n^{5 / 4}} \int_{0}^{2(n-1)} \widetilde{R}_{n}(t) \mathrm{d} t=\frac{1}{2 n^{5 / 4}} \int_{0}^{2(n-1)} R_{n}(t) d t+O\left(n^{-1 / 4}\right)=\int_{0}^{1} r_{n}(s) d s+o(1),
$$

where $r_{n}(s) \stackrel{\text { def }}{=} n^{-1 / 4} R_{n}(2(n-1) s)$. Also normalize $v_{n}(s) \stackrel{\text { def }}{=} n^{-1 / 2} V_{n}(2(n-1) s)$. Theorem 2 of [14] (see also [10]) states that $\left(r_{n}, v_{n}\right) \stackrel{\mathrm{d}}{\longrightarrow}(r, v)$ in $C[0,1] \times C[0,1]$, with $r, v$ to be defined shortly.

Before defining $r$ and $v$, we will briefly motivate what they ought to be. Firstly, as the offspring distribution $\xi$ of $T_{n}$ satisfies $\mathbb{E}[\xi]=1$, we expect the tour $V_{n}$ to be roughly a random walk with zero-mean increments, conditioned to be non-negative and return to the origin at time $2(n-1)$, and the limiting law $v$ ought to be a Brownian excursion (up to a constant scale factor). Secondly, consider a node $u$ and the path $\rho=u_{0}, u_{1} \ldots, u_{d}=u$, where $d$ is the depth of $u$. We can define a random walk $\Phi_{u}(t)$ for $t=0, \ldots, d$ by $\Phi_{u}(0)=0$ and $\Phi_{u}(t)=\sum_{i=1}^{t} Q_{u_{i}}$ for $t>0$, noting that $\Phi_{u}=\Phi_{u}(d)$. Under rescaling, the random walk $\Phi_{u}(t)$ will behave like Brownian motion. For any two nodes $u_{1}, u_{2}$ with last common ancestor at depth $m$, the processes $\Phi_{u_{1}}, \Phi_{u_{2}}$ agree for $t=0, \ldots, m$, while any subsequent increments are independent. Hence $\operatorname{Cov}\left(\Phi_{u_{1}}, \Phi_{u_{2}}\right)=\mathrm{cm}$ for some constant $c>0$. Now, for any $i, j \in\{0, \ldots, 2(n-1)\}$, the nodes $f(i), f(j)$ at depths $V_{n}(i), V_{n}(j)$ have last common ancestor $f(k)$, where $k$ is such that $V_{n}(k)$ is minimal in the range $i \leq k \leq j$. Hence $r(s)$ should be normally distributed with variance given by $v(s)$, and the covariance of $r(s), r(t)$ proportional to $\min _{s \leq u \leq t} v(u)$.

We now define $r, v$ precisely. If $\operatorname{Var} \xi=\sigma^{2}$, then $v(s) \stackrel{\text { def }}{=} 2 \sigma^{-1} e(s)$, where $e(s)$ is a standard Brownian excursion, as shown by Aldous [1, 2]. Conditioning on $v$, we define $r$ as a centered 
Gaussian process on $[0,1]$ with

$$
\operatorname{Cov}(r(s), r(t) \mid v)=\frac{1}{12} \min _{s \leq u \leq t} v(u)=\frac{1}{12 \sigma} C(s, t), \quad s \leq t .
$$

The constant $1 / 12$ appears as the variance of the random increments $Q_{v}$. Again, Theorem 2 of [14] states that $\left(r_{n}, v_{n}\right) \stackrel{\mathrm{d}}{\longrightarrow}(r, v)$ in $C[0,1]^{2}$. We conclude that

$$
\lim _{n \rightarrow \infty} \frac{J\left(T_{n}\right)}{n^{5 / 4}}=\int_{0}^{1} r_{n}(t) d t+o(1) \stackrel{\mathrm{d}}{\longrightarrow} \int_{0}^{1} r(t) d t \stackrel{\text { def }}{=} Y .
$$

This integral is the object of study in [13], wherein it is shown that

$$
Y \stackrel{\text { def }}{=} \int_{0}^{1} r(t) d t \stackrel{\mathrm{d}}{=} \frac{1}{\sqrt{12 \sigma}} \sqrt{\eta} \mathcal{N}
$$

where $\mathcal{N}$ is a standard normal variable, $\eta$ is given by

$$
\eta=\int_{[0,1]^{2}} C(s, t) \mathrm{d} s \mathrm{~d} t,
$$

and $\eta, \mathcal{N}$ are independent. The odd moments of $Y$ are zero, as this is the case for $\mathcal{N}$, and by $[13$, Theorem 1.1], for $k \geq 0$

$$
\mathbb{E}\left[Y^{2 k}\right]=\frac{1}{(12 \sigma)^{k}} \frac{(2 k !) \sqrt{\pi}}{2^{(9 k-4) / 2} \Gamma((5 k-1) / 2)} a_{k},
$$

where $a_{1}=1$ and for $k \geq 2$,

$$
a_{k}=2(5 k-4)(5 k-6) a_{k-1}+\sum_{i=1}^{k-1} a_{i} a_{k-i} .
$$

In particular ([13, Theorem 1.2]),

$$
\mathbb{E}\left[Y^{2 k}\right] \sim \frac{1}{(12 \sigma)^{k}} \frac{2 \pi^{3 / 2} \beta}{5}(2 k)^{1 / 2}\left(10 e^{3}\right)^{-2 k / 4}(2 k)^{\frac{3}{4} \cdot 2 k},
$$

as $k \rightarrow \infty$, where $\beta=0.981038 \ldots$ Further analysis of the moments of $\eta$ and $Y$, including the moment generating function and tail estimates, can be found in [13].

The last bit of Theorem 6 which remains to be proved is that $\mathbb{E}\left[e^{t Y_{n}}\right] \rightarrow \mathbb{E}\left[e^{t Y}\right]$ for all fixed $t \in \mathbb{R}$. Since we have already shown $Y_{n} \stackrel{\mathrm{d}}{\longrightarrow} Y$, we can apply the Vitali convergence theorem once we have shown that the sequence $e^{t Y_{n}}$ is uniformly integrable. See Section 5.1 of [4] for details.

\section{References}

1 David Aldous. The continuum random tree. II. An overview. In Stochastic analysis (Durham, 1990), volume 167 of London Math. Soc. Lecture Note Ser., pages 23-70. Cambridge Univ. Press, Cambridge, 1991.

2 David Aldous. The continuum random tree. III. Ann. Probab., 21(1):248-289, 1993.

3 Nicolas Broutin and Cecilia Holmgren. The total path length of split trees. Ann. Appl. Probab., 22(5):1745-1777, 2012.

4 Xing Shi Cai, C. Holmgren, S. Janson, T. Johansson, and F. Skerman. Inversions in split trees and conditional Galton-Watson trees. ArXiv e-prints, 2017. arXiv:1709.00216. 
5 G. Cramer. Introduction à l'analyse des lignes courbes algébriques. Cramer, 1750.

6 Luc Devroye. Universal limit laws for depths in random trees. SIAM J. Comput., 28(2):409432, 1999.

7 William Feller. An introduction to probability theory and its applications. Vol. I. John Wiley \& Sons, Inc., New York-London-Sydney, 3rd edition, 1968.

8 P. Flajolet, P. Poblete, and A. Viola. On the analysis of linear probing hashing. Algorithmica, 22(4):490-515, 1998.

9 Ira M. Gessel, Bruce E. Sagan, and Yeong Nan Yeh. Enumeration of trees by inversions. J. Graph Theory, 19(4):435-459, 1995.

10 Bernhard Gittenberger. A note on: "State spaces of the snake and its tour-convergence of the discrete snake". J. Theoret. Probab., 16(4):1063-1067 (2004), 2003.

11 Svante Janson. The Wiener index of simply generated random trees. Random Structures Algorithms, 22(4):337-358, 2003.

12 Svante Janson. Simply generated trees, conditioned Galton-Watson trees, random allocations and condensation: extended abstract. In 23rd Intern. Meeting on Probabilistic, Combinatorial, and Asymptotic Methods for the Analysis of Algorithms (AofA'12), Discrete Math. Theor. Comput. Sci. Proc., AQ, pages 479-490. Assoc. Discrete Math. Theor. Comput. Sci., Nancy, 2012.

13 Svante Janson and Philippe Chassaing. The center of mass of the ISE and the Wiener index of trees. Electron. Comm. Probab., 9:178-187, 2004.

14 Svante Janson and Jean-François Marckert. Convergence of discrete snakes. J. Theoret. Probab., 18(3):615-647, 2005.

15 Donald E. Knuth. The art of computer programming. Vol. 3. Addison-Wesley, Reading, MA, 1998.

16 C. L. Mallows and John Riordan. The inversion enumerator for labeled trees. Bull. Amer. Math. Soc., 74:92-94, 1968.

17 Barbara H. Margolius. Permutations with inversions. J. Integer Seq., 4(2):Article 01.2.4, $13,2001$.

18 Ralph Neininger. On a multivariate contraction method for random recursive structures with applications to Quicksort. Random Structures Algorithms, 19(3-4):498-524, 2001. Analysis of algorithms (Krynica Morska, 2000).

19 Ralph Neininger and Ludger Rüschendorf. On the internal path length of $d$-dimensional quad trees. Random Structures Algorithms, 15(1):25-41, 1999.

20 Alois Panholzer and Georg Seitz. Limiting distributions for the number of inversions in labelled tree families. Ann. Comb., 16(4):847-870, 2012.

21 Uwe Rösler. A limit theorem for "Quicksort". RAIRO Inform. Théor. Appl., 25(1):85-100, 1991.

22 Vladimir N. Sachkov. Probabilistic methods in combinatorial analysis, volume 56 of Encyclopedia of Mathematics and its Applications. Cambridge University Press, Cambridge, 1997. Translated from the Russian, Revised by the author. 